\title{
Thoracic X-Strap Harness. Design and Method 2
}

Duncan Orr-Ewing, David Anderson \& Ewan Weston

http://dx.doi.org/10.4314/vulnew.v78ai1.6

This method has been used on Golden Eagles Aquila chrysaetos and White-tailed Eagles Haliaeetus albicilla in Scotland and on Gyps vultures in Asia.

\section{Equipment needed:}

- Ornitela or Microwave Telemetry 30g satellite tag

- Teflon ribbon: $11.2 \mathrm{~mm}$ wide (0.44”)

- Silicone tubing four pieces, $3 \mathrm{~mm}$ width, $110 \mathrm{~mm}$ length

- Prepared circular breast template made from a PVC milk bottle or similar material

- Linen thread (Hemline or similar, available from Amazon)

- Surgical clamps (UK NHS or similar), bulldog clips

- Small sharp scissors

- Curved needles (available from Amazon)

- Superglue/leather glue (Bostik)

- Neoprene foam material (available on Amazon)

- Marker pen

The satellite tag and harness is largely prepared in advance.

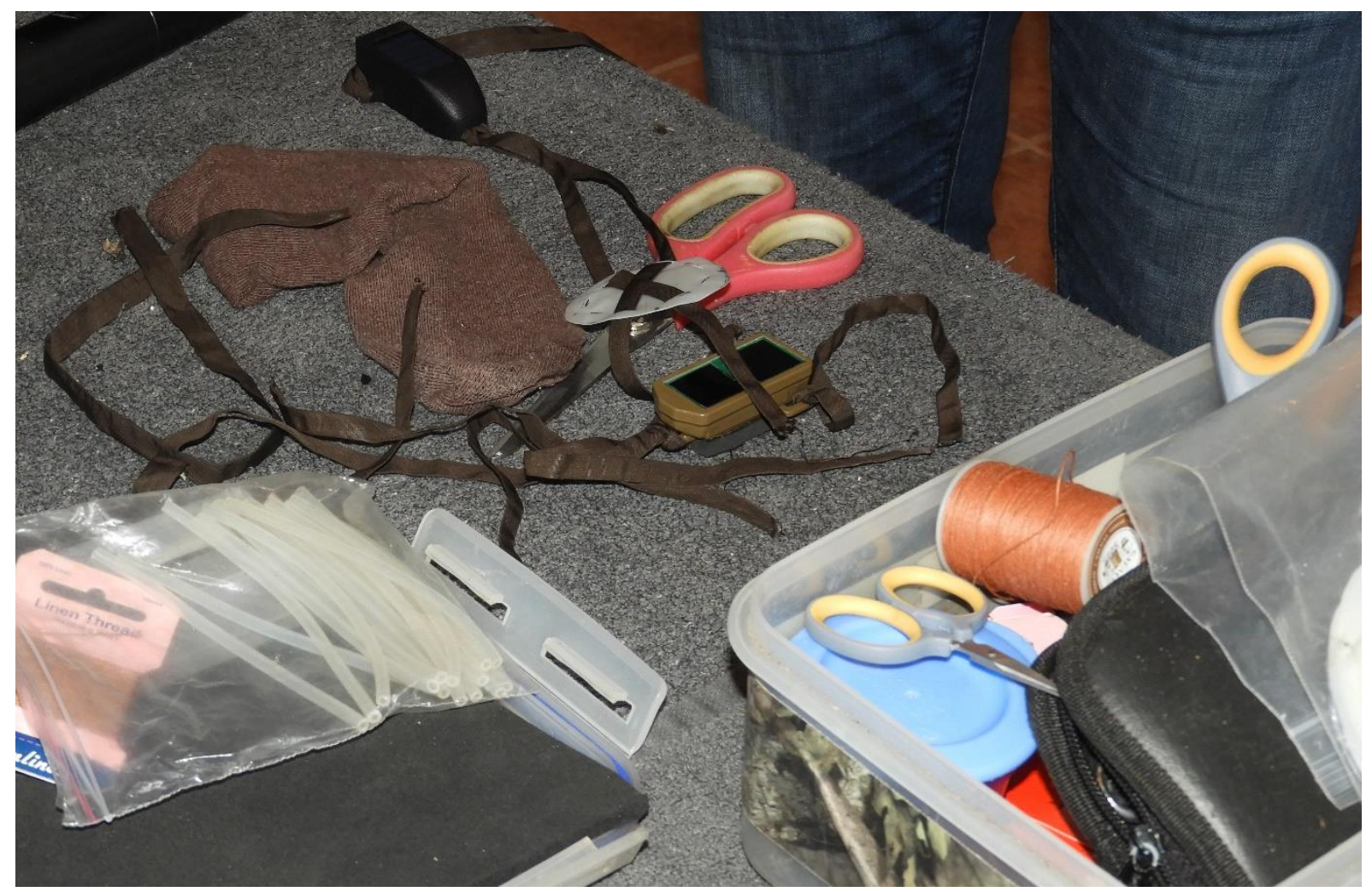

Figure 2.1: Equipment and materials. 


\section{Measurements and preparations: Key stages/steps}

1) Take two pieces of Teflon ribbon. Cut lengths (for White-rumped Vulture- $660 \mathrm{~mm}$ for the back ribbon and $620 \mathrm{~mm}$ for the front ribbon).

2) For Ornitela or Microwave Telemetry $30 \mathrm{~g}$ tags, pass $620 \mathrm{~mm}$ ribbon through the front attachment lug at the front of the device using clamps to pull through as required. Tie a simple loop knot at the centre and over the lug making sure the loose strands are of equal length. Knot can be lightly superglued as required. Ornitela tag requires attachment of neoprene foam base using leather glue (Bostik). The surface area of the neoprene base should be wide enough to cover whole tag including lugs.

3) Take $660 \mathrm{~mm}$ ribbon and pass through the two attachment lugs at the back of the device tying a loop knot in each and passing ribbon either under or around the back of the tag, checking that the two strands are of equal length. No sewing needed for these knots, however knot can also be lightly superglued as required.

4) Cut four pieces of hollow silicone tubing to $110 \mathrm{~mm}$ lengths and cover in talcum powder to help push them into tubular Teflon ribbon. Tubing stays in place in tag although does not pass through lug and is short enough to leave room at end with no tubing in ribbon to push through breast template and for sewing later at sternum.

5) Using a thin plastic sheet or other durable material (e.g. UK plastic milk bottles) to make round circular discs $50 \mathrm{~mm}$ in diameter. Cut out eight slits with sharp scissors around the disc (see images below), big enough through which tubular Teflon ribbon can be passed.

6) Fit front two straps through the top two slits of the template and diagonally across to bottom two slits so ribbons cross in the middle of the template.

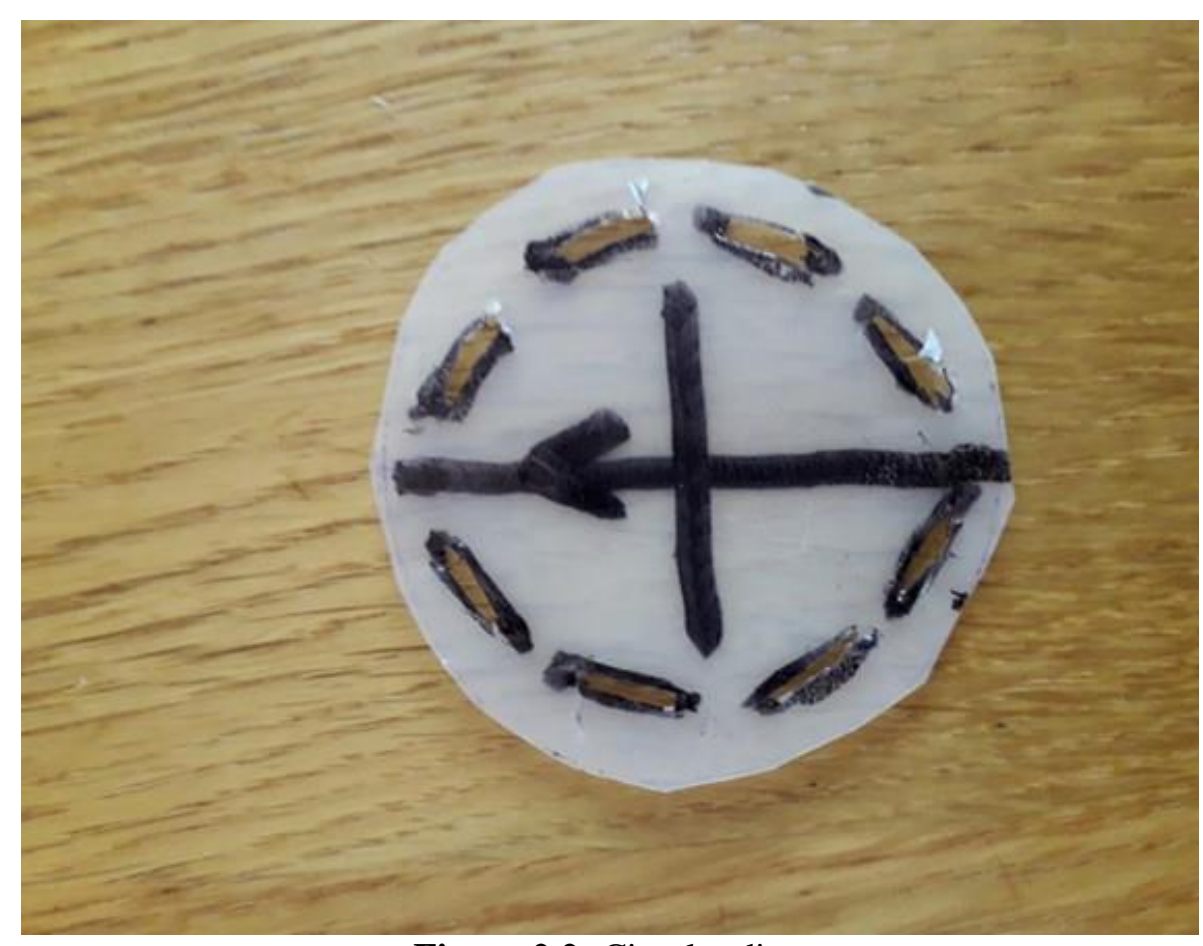

Figure 2.2: Circular disc. 


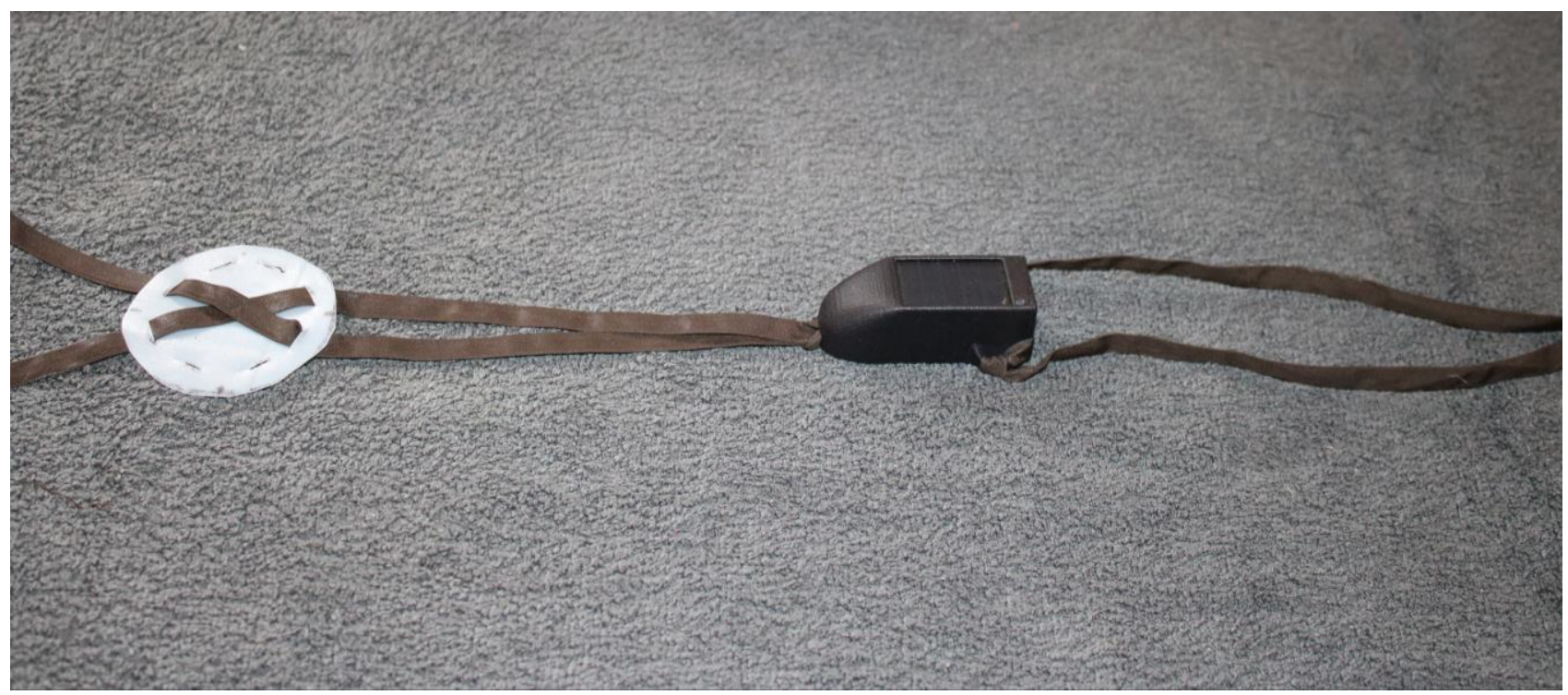

Figure 2.3: Prepared harness.

\section{Restraint and hooding}

Using this method, the tag is best fitted by one person holding the vulture upright whilst the person holding the bird is leaning against a table or similar. The holder of the bird will have one hand around the legs of the bird with finger between legs and another holding the neck below the head of the bird. The breast of the of the bird should face forward. A falconry hood should be fitted to keep the bird calm. The other person then fits the tag. An experienced holder will be able to help move the bird to allow ribbons to be easily passed under wings and manoeuvre the bird into the right positions for fitting the tag. This work is therefore best done by two experienced practitioners.

\section{Fitting to the bird}

1) Pass the front two straps attached to the harness over the head of the bird. Ensure tag is placed on upper back of the bird where it should sit, and adjust template to align with the sternum avoiding the crop. Then bring back two straps over and under wing, carefully ensuring that no wing feathers are caught up in the harness lug , and thread diagonally through remaining slits on template. All harness ribbons should now cross at the centre of the template at sternum. Ensure that all ribbons are not twisted. Clamp in place using surgical clamps or bulldog clips. Then preen ribbons into place to ensure that ribbons are next to the skin of the bird. Using clamps to hold ribbons in place adjust ribbons to ensure good fit on bird allowing two index fingers to fit under tag on back of bird. Ensure that front and back ribbons are each the same length.

2) When the tag is correctly adjusted take a length of linen twine and a curved needle and sew through the middle of the four ribbons where they cross at the sternum, starting from the top of the ribbons to ensure no knots are on the underside of the harness and adjacent to the skin of the bird. The presence of the template ensures that you cannot sew the skin or feathers of the bird. Sew through the middle of the four ribbons 5-6 times; then seal the knot by sewing through it and binding it on the top side; and then remove the clamps. Check all four ribbons are correctly sewn together. Then carefully cut off the template using sharp scissors, avoiding cutting the harness. 


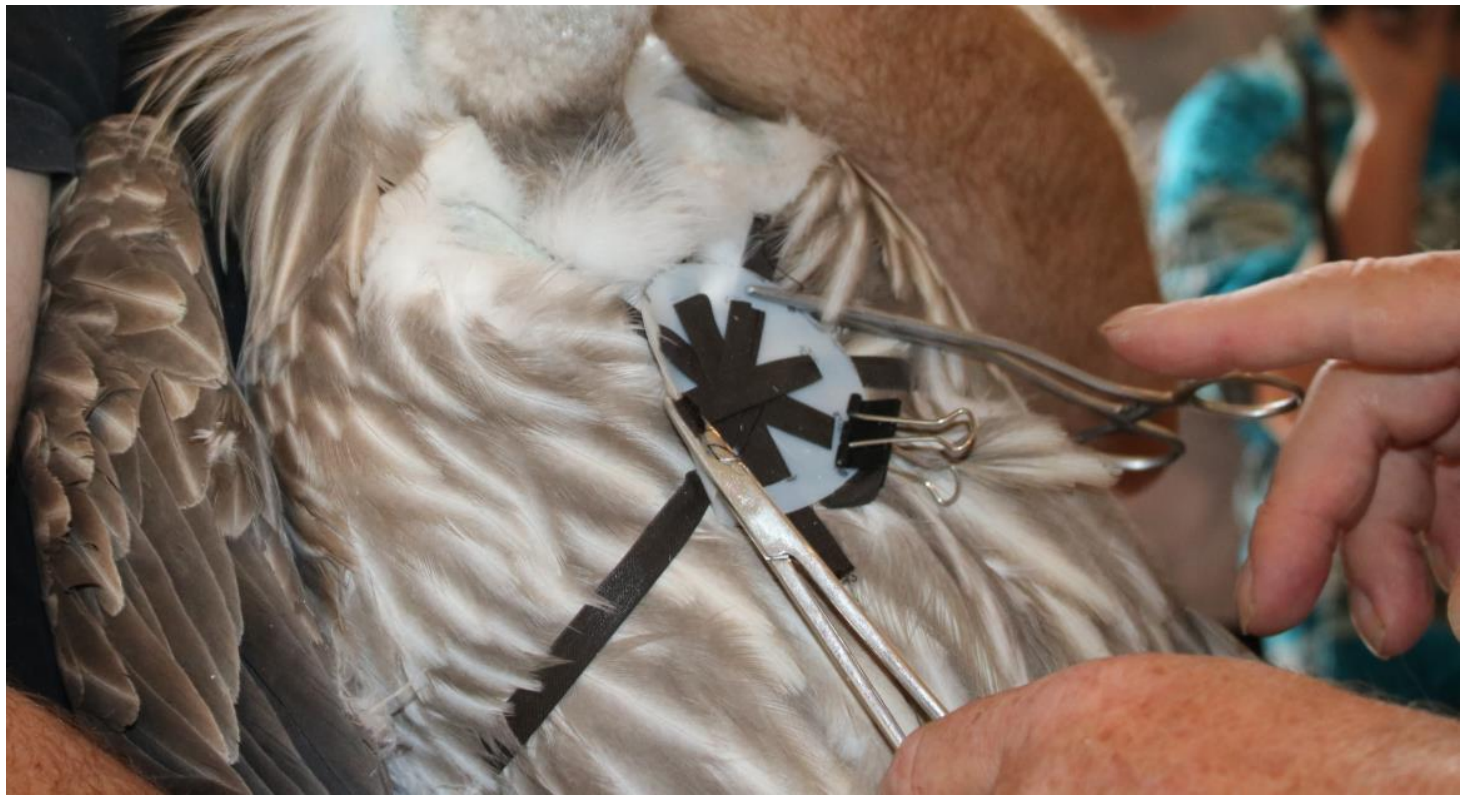

Fig. 2.4: Clamping the harness in place.

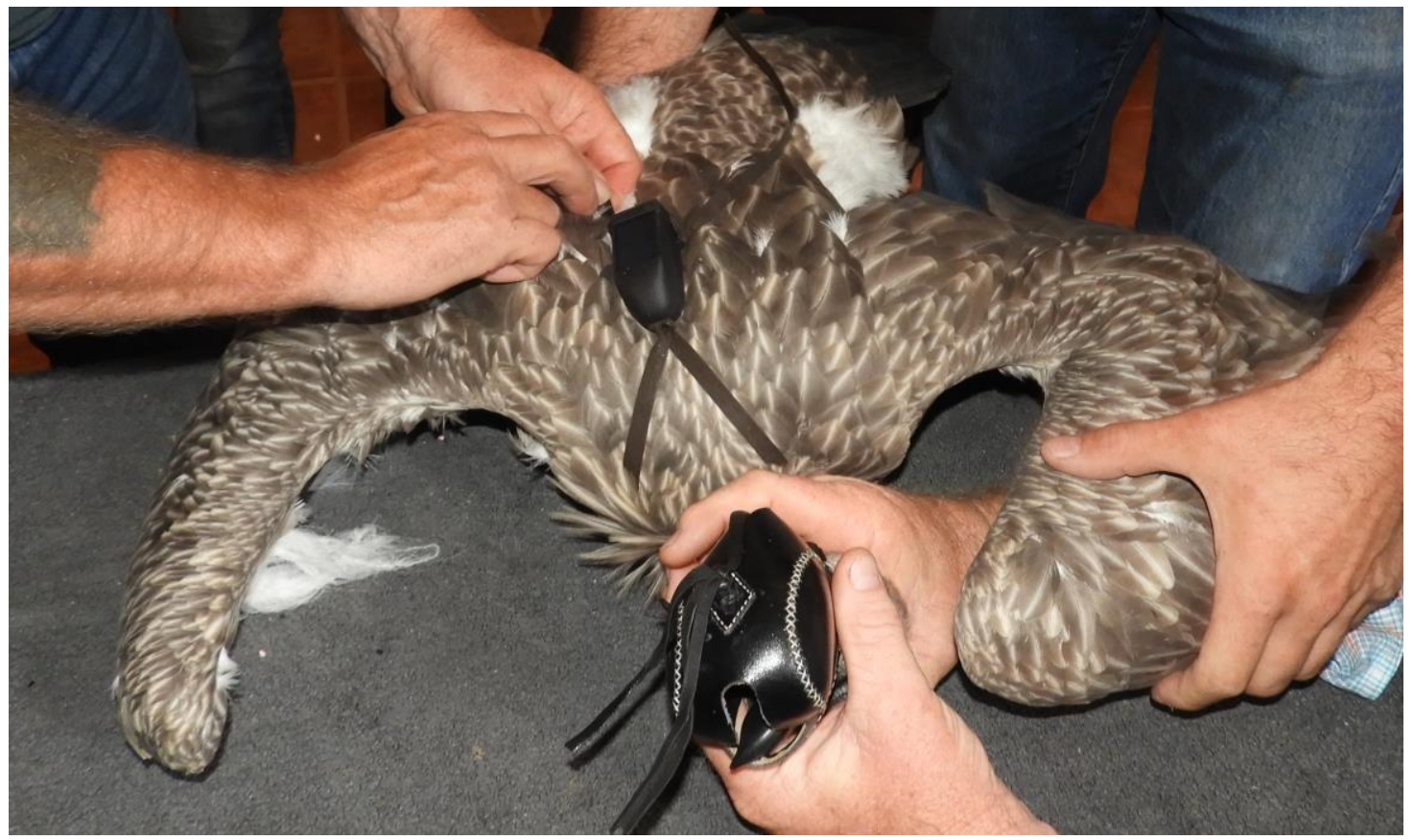

Figure 2.5: Securing the harness.

3) Check the harness and fit of the tag on the bird again, by running your fingers around the Teflon loops and putting index fingers under the tag. Use a small amount of Superglue to seal the knot on the sternum.
Cut the four ends of Teflon ribbon at the breast template to make them shorter and then seal each Teflon ribbon at the end with a strip of $5 \mathrm{~mm}$ of Superglue to prevent unravelling. 